Arch Virol (1994) [Suppl] 9: 173-180

Archives

Virrology

(C) Springer-Verlag 1994

\title{
Role of subgenomic minus-strand RNA in coronavirus replication
}

\author{
D. A. Brian, R.-Y. Chang, M. A. Hofmann, and P. B. Sethna \\ Department of Microbiology, University of Tennessee, Knoxville, Tennessee, U.S.A.
}

Summary. Coronavirus subgenomic minus-strand RNAs (negative-strand copies of the $3^{\prime}$ coterminal subgenomic mRNAs) probably function in mRNA amplification by serving as templates for transcription from internal (intergenic) promoters, rather than by faithful (full-length) mRNA replication.

\section{Introduction}

Coronaviruses replicate by generating a $3^{\prime}$ coterminal nested set of 6 to 8 subgenomic mRNA species (reviewed in [1]). When it was first reported that each subgenomic mRNA also had a minus-strand counterpart [2], it was hypothesized that perhaps each mRNA species underwent faithful replication by using the same mechanism(s) as the genome. This hypothesis was based on three sets of observations and an assumption: (i) Coronavirus subgenomic RNA species (both plus and minus strands) underwent amplification early in infection at a rate that was inversely related to their length. (ii) Subgenomic replicative forms existed. (iii) Each subgenomic mRNA species possessed in common with the genome a $3^{\prime}$ terminal sequence of approximately 300 nucleotides (excluding the poly(A) tail) and a $5^{\prime}$ terminal sequence (a leader) of 65 to 90 nucleotides (the length depends on the species of coronavirus). The assumption was that these termini possessed respectively the promoter and the promoter template for minus- and plus-strand RNA synthesis. More recent reports showing mRNA amplification in other coronavirus species [3, 4], a structural analysis of subgenomic replicative forms [4], and the existence of an antileader on the $3^{\prime}$ end of subgenomic minusstrand RNAs [5], in our view supported the notion that mRNAs undergo replication.

To directly test the hypothesis that coronavirus subgenomic mRNAs do undergo faithful replication, clones of mRNA species containing a 
reporter sequence were used to make transcripts in vitro that were then transfected into infected cells, and evidence of replication was sought.

To date we have found no direct evidence of mRNA replication following transfection of marked transcripts (synthetic mRNAs) into infected cells. These results, along with (i) the successful demonstration that the cloned 5 '-terminal $498 \mathrm{nt}$ fragment of a defective-interfering RNA species imparts replicational competence when spliced onto the $5^{\prime}$ end of a marked mRNA molecule (described below), and, (ii) the demonstration that a sub-defective-interfering RNA transcript can be generated from a cloned defective-interfering RNA after insertion of a coronavirus intergenic sequence [6], lead us to conclude that perhaps coronavirus subgenomic mRNAs do not amplify by a faithful replication mechanism. Rather, they may amplify by a transcriptional mechanism in which the subgenomic minus-strand templates function by an internal promoter to make new mRNA of smaller size, but not of equal size.

\section{The antileader on subgenomic minus-strand RNA is apparently an insufficient promoter for plus-strand synthesis (at least when the template mRNA enters via transfection)}

To determine whether subgenomic mRNA molecules can undergo replication following transfection into infected cells, cloned mRNA for the $\mathrm{N}$ proteins of porcine transmissible gastroenteritis virus (TGEV) and bovine coronavirus $(\mathrm{BCV})$ were modified to contain a reporter sequence (either the entire CAT gene or a small portion of it were cloned in-frame within the N ORF) and in vitro generated transcripts were transfected into infected cells and evidence of amplified plus- and minus-strands were sought. In several experiments, no evidence of amplification (i.e., replication) was found (P. Sethna and M. Hofmann, unpubl. data). In one such experiment (Fig. 1), the BCV N mRNA with a $30 \mathrm{nt}$, inframe, reporter sequence (a $30 \mathrm{nt}$ fragment of the TGEV N ORF, chosen because it would be unlikely to have a detrimental effect on the stability of the molecule) was used for transfection into BCV-infected cells. Whereas the mRNA species and genome of helper BCV can be seen to increase in amount after infection and transfection (Fig. 1B, lanes 2 through 5), input mRNA transcript carrying a reporter sequence (Fig. 1B, lane 6) did not increase (lanes 7-10). When reporter minusstrand-detecting probe was used, no minus-strand RNA was detected at all after transfection (Fig. 1B, lanes 12-15). Thus, the transfected transcript did not undergo replication.

It is important to note that even though minus-strand RNAs were not sufficiently abundant to be detected in the RNA blotting experiment described in Fig. 1B, we assume they were made in small numbers after 


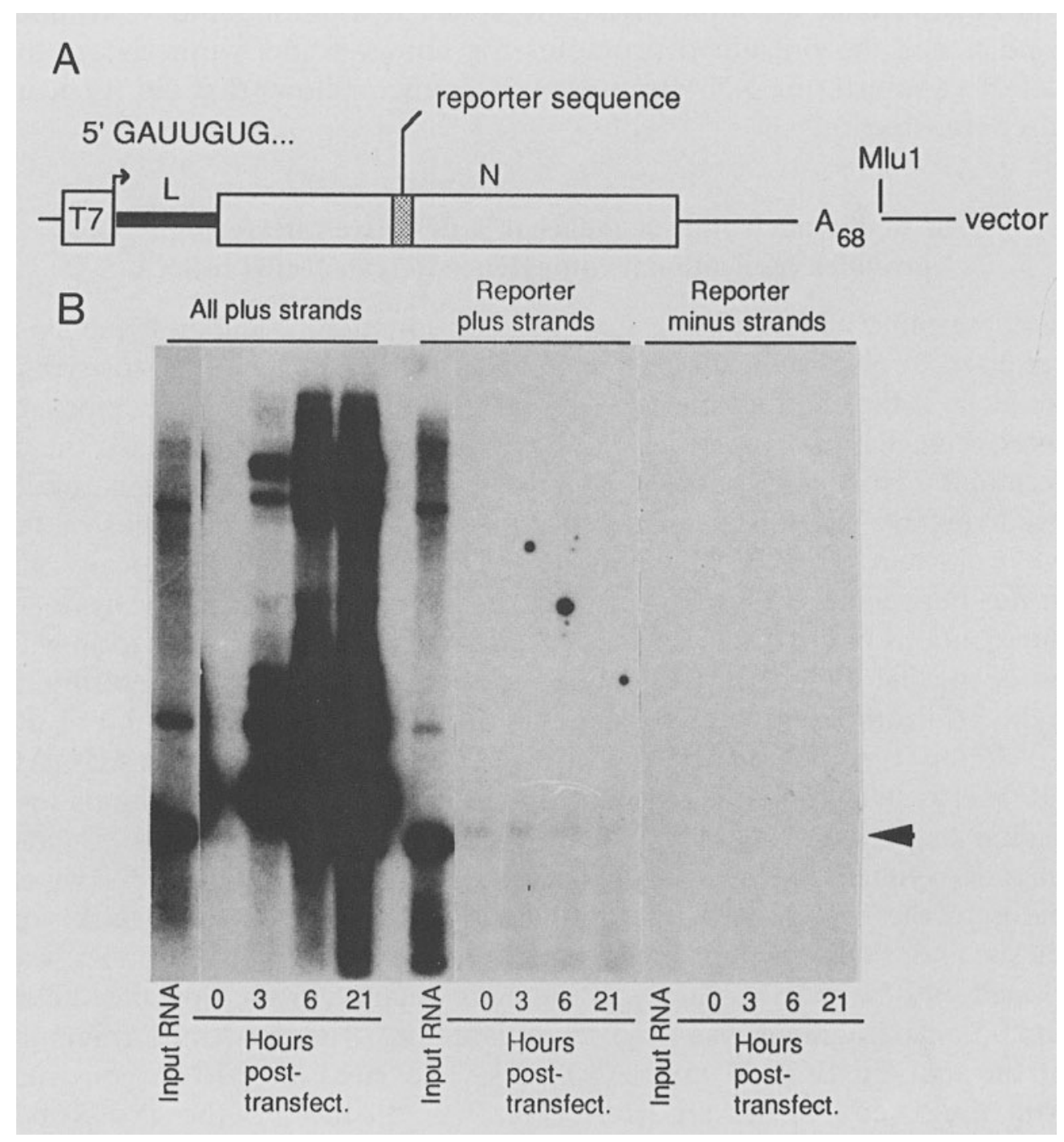

Fig. 1. A Plasmid construct of the cloned bovine coronavirus $\mathrm{N}$ mRNA with a 30 nucleotide, in-frame, reporter sequence. Plasmid cut with Mlu1 and transcribed with T7 RNA polymerase generates transcripts beginning with a $5^{\prime}$ GAUUGUG..., (the first bases in the 65 nucleotide leader [L]), and $\mathrm{N}$ open reading frame of 478 nucleotides (including the reporter), and a poly A tail of 68 nucleotides. B RNA blot (Northern) showing successful transfection of reporter-containing $\mathrm{N}$ mRNA, but no replication. HRT-18 cells were infected with the bovine coronavirus $(\mathrm{MOI}=5)$ for $1 \mathrm{~h}$, then transfected with transcripts $(200 \mathrm{ng} / 35 \mathrm{~mm}$ plate) for $1 \mathrm{~h}$. Cytoplasmic RNA was extracted at the times indicated, electrophoresed in a formaldehyde-agarose gel, transferred to a nylon membrane, and probed with ${ }^{32} \mathrm{P}$-end-labeled oligonucleotide. An oligonucleotide hybridizing to $\mathrm{N}$ plus strands was used to identify all viral RNA species, one hybridizing to reporter plus strands was used to identify reporter-containing plus strands, and one hybridizing to reporter minus strands was used to identify reportercontaining minus strands. Note no increase in the amount of reporter-containing plus strands and the absence of reporter-containing minus strands 
transfection since the input mRNA template shared the same $3^{\prime}$ terminus (and hence the presumed promoter for minus-strand synthesis) as the defective-interfering RNA transcript (described below) that did replicate after transfection.

\section{The 5' 498 nucleotide sequence of a defective-interfering RNA provides replicational competence to transfected mRNA}

To determine whether more than just the common $5^{\prime}$ leader sequence is required to give replicational competence to the subgenomic plus-strand molecule, the $5^{\prime}$ terminal $498 \mathrm{nt}$ of a naturally occurring defectiveinterfering RNA of $\mathrm{BCV}$ was cDNA cloned and used to replace the $5^{\prime}$ terminal $77 \mathrm{nt}$ of the marked BCV N mRNA (Fig. 2A). The naturally occurring BCV DI RNA is present in one of our cloned stocks of the Mebus strain of BCV [3]. The BCV DI RNA electrophoretically migrates between the $\mathrm{N}$ and $\mathrm{M}$ mRNA species on a denaturing agarose gel and contains N ORF sequence as determined by RNA hybridization with an N-specific oligodeoxynucleotide, and leader sequence as determined by hybridization with a leader-specific probe. To clone the $5^{\prime}$ end of the DI RNA, oligodeoxynucleotide 5'CCAGAACGATTTCCAAAGGAC GCTCT5', which contains an internal unique Xmn1 site and binds to a region beginning $34 \mathrm{nt}$ downstream from the start of N ORF [7], and oligodeoxynucleotide 5'GATTGTGAGCGATTTGCGTGCG3', which binds to the first $22 \mathrm{nt}$ of the antileader [8], were used for cDNA synthesis and PCR amplification, and the amplified $557 \mathrm{nt}$ product was cloned into a pGEM3Zf(-) (Promega) vector. From this the $499 \mathrm{nt}$ $\mathrm{Bgl} 2-\mathrm{Xmn} 1$ fragment was used to replace the $78 \mathrm{nt} \mathrm{Bgl}-\mathrm{Xmn} 1$ fragment of the marked BCV N mRNA (Fig. 1A) to yield an mRNA construct with the $5^{\prime}$ end of the DI RNA (Fig. 2A). In the DI, the $5^{\prime} 498 \mathrm{nt}$ is presumed to represent the $5^{\prime}$ end of the $\mathrm{BCV}$ genome since it shows extremely high sequence similarity to the $5^{\prime}$ end of the MHV genome. In the DI, the 5' DI-specific ORF of 96 amino acids (hatched line in Fig. $2 \mathrm{~A}$ ) is contiguous with the N ORF.

Transcripts of the marked $\mathrm{N}$ mRNA containing the 5' DI RNAspecific sequence underwent replication as determined by an increase in plus-strand accumulation (Fig. 2B, lanes 13-14) and the appearance of minus-strand species (Fig. 2B, lane 20).

\section{Possible mechanisms of mRNA amplification from subgenomic minus strands}

The rapid appearance of coronavirus subgenomic mRNA species and the abundance of transcriptionally active subgenomic replicative inter- 


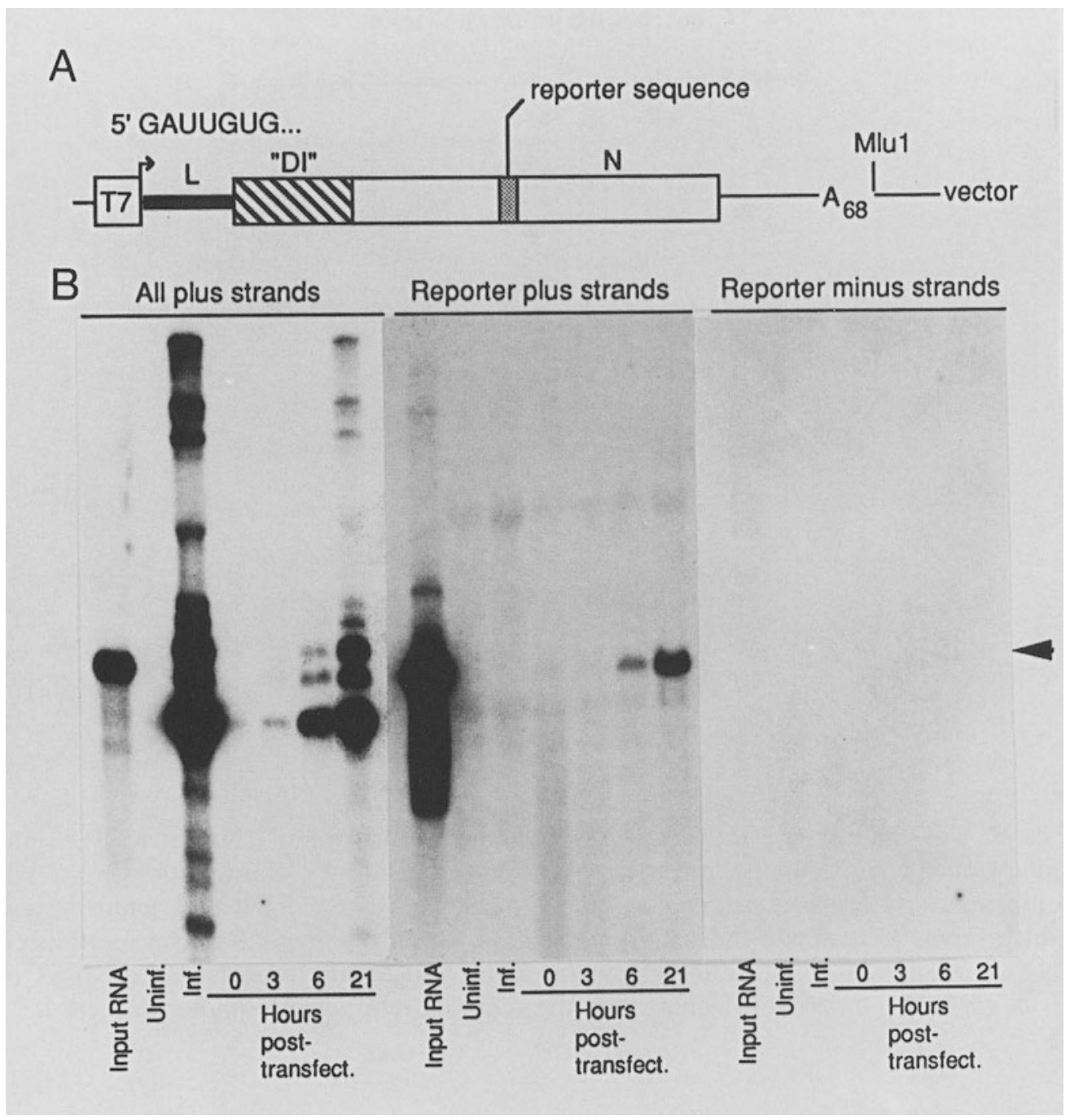

Fig. 2. A Plasmid construct of the cloned bovine coronavirus defective-interfering RNA with a 30 nucleotide, in-frame, reporter sequence. The construct is identical to that described for the $\mathrm{N}$ mRNA in Fig. 1A, except for 433 nucleotides of DI-specific sequence inserted between the $65 \mathrm{nt}$ leader and the start codon of N. 288 of the $433 \mathrm{nt}$ make up a DI-specific open reading frame that is in-frame with the $\mathrm{N}$ open reading frame. B RNA blot (Northern) analysis performed as described in Fig. 1B

mediates suggest that subgenomic minus-strand molecules are important in the process of mRNA amplification.

We envision two possible mechanisms for the amplification of mRNA from subgenomic minus strands. The first is illustrated in Fig. 3A in which the subgenomic mRNA arises, perhaps by a leader priming mechanism, from the antigenomic template (as illustrated) or perhaps by 

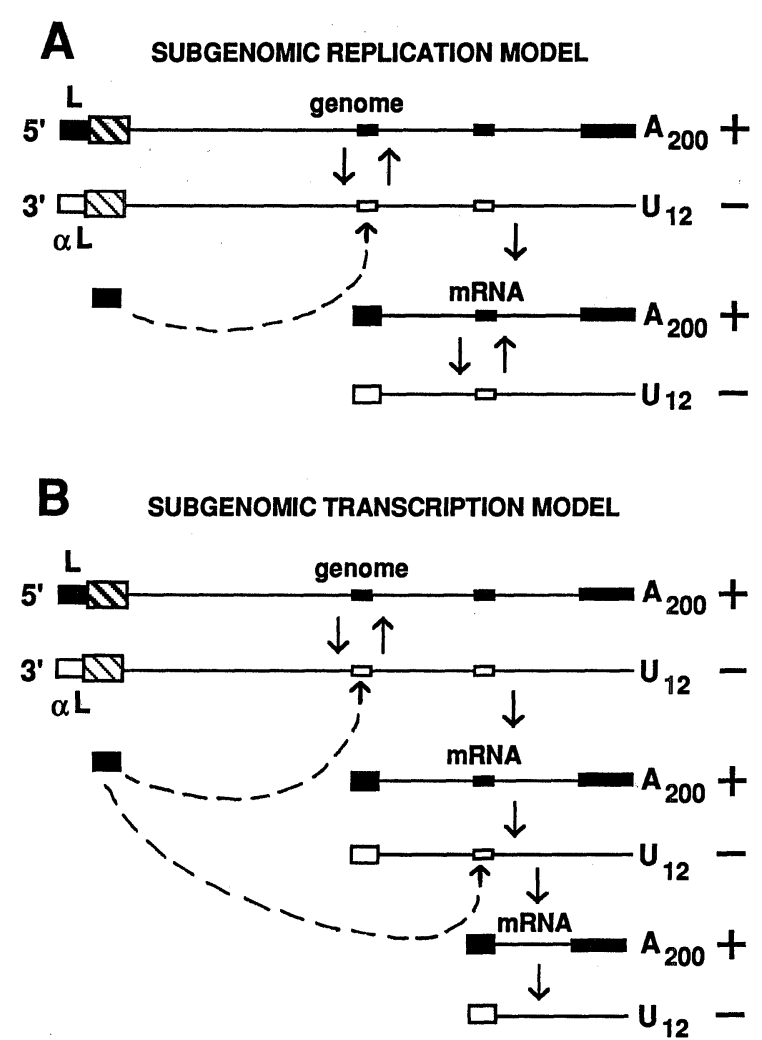

Fig. 3. Two models for the role of subgenomic minus-strand RNA in mRNA amplification. A Subgenomic mRNA (produced originally by transcription from the antigenome by a leader-priming or other mechanism) gets copied to a minus strand which serves as template for mRNA replication. B Subgenomic mRNAs are produced by the usual transcription mechanism from the antigenome or from antimRNAs of larger size (i.e., those which contain the requisite intergenic promoter sequence)

splicing of the genome or antigenome. Once made, the mRNA (or antimRNA) serves as template for faithful replication using the same mechanism as genome. We interpret the failure of synthetic mRNAs to undergo replication after transfection in the above described experiments to mean either one of two things: (i) mRNAs do not replicate. (ii) mRNAs replicate, but require a special juxtapositional start within the replicational machinery that is gained from the original transcriptional process. That is, mRNA transfected into the cell cannot initiate a cycle of replication without the added cis-acting sequence provided by the genomic (i.e., DI) $5^{\prime}$ end. Further experimentation is required to distinguish between these two possibilities.

The second potential mechanism for amplification of mRNA is illustrated in Fig. 3B. In this case, minus-strand RNAs that are synthesized from either genome or subgenomic mRNAs (generated by 
leader-priming or splicing as described above) can serve as templates for new plus-strand synthesis, but by a leader priming (or splicing) step, not by replication. By this mechanism, subgenomic minus-strand RNA would serve as a template for synthesis of mRNA of smaller size, but not of the same size. Evidence supporting this mechanism comes from the laboratory of Sinji Makino (University of Texas, Austin) in which subDI RNA transcripts were generated from a transfected, replicating DI RNA construct into which the conserved UCUAAAC intergenic sequence element (and some flanking sequence) had been internally placed [6]. Presumably this sequence element is functioning on the minus strand to direct priming (or splicing) to generate the subgenomic (sub DI) transcript. With this second mechanism, a subgenomic minus-strand RNA without an antileader could also theoretically function as a template for transcription.

\section{Conclusions}

Coronaviruses appear unique among positive strand RNA viruses in that subgenomic minus-strand RNAs are important as templates for the amplification of subgenomic mRNA species. Subgenomic minus-strand RNAs apparently serve as templates for greater than $90 \%$ of subgenomic mRNA synthesis (Stan Sawicki, Medical College of Ohio, Toledo, Ohio, personal communication). It remains to be determined, however, whether, subgenomic mRNAs amplify by (i) mRNA replication, (ii) transcription by internal initiation on a subgenomic minus strand template, or (iii) both. At the present time, our data favor the second option.

\section{References}

1. Lai MMC (1990) Coronavirus: organization, replication and expression of genome. Annu Rev Microbiol 44: 303-333

2. Sethna PB, Hung SL, Brian DA (1989) Coronavirus subgenomic minus-strand RNAs and the potential for mRNA replicons. Proc Natl Acad Sci USA 86: 5626-5630

3. Hofmann MA, Sethna PB, Brian DA (1990) Bovine coronavirus mRNA replication continues throughout persistent infection in cell culture. J Virol 64: 4108-4114

4. Sawicki SG, Sawicki DL (1990) Coronavirus transcription: subgenomic mouse hepatitis virus replicative intermediates function in mRNA synthesis. J Virol 64: $1050-1056$

5. Sethna PB, Hofmann MA, Brian DA (1991) Minus-strand copies of replicating coronavirus mRNAs contain antileaders. J Virol 65: 320-325

6. Makino S, Myungsoo J, Makino JK (1991) A system for study of coronavirus mRNA synthesis: a regulated, expressed subgenomic defective interfering RNA results from intergenic site insertion. J Virol 65: 6031-6041

7. Lapps W, Hogue BG, Brian DA (1987) Sequence analysis of the bovine coronavirus nucleocapsid and matrix protein genes. Virology 157: 47-57 
8. Senanayake SD, Hofmann MA, Maki JL, Brian DA (1992) The nucleocapsid protein gene of the bovine coronavirus is bicistronic. J Virol 66: 5277-5283

Authors' address: Dr. D. A. Brian, Department of Microbiology, University of Tennessee, Knoxville, TN 37996-0845, U.S.A. 